\title{
An Open Forensic Unit for the Borderline Mentally Impaired Offender
}

Jeanette Smith, Registrar, Wonford House Hospital, Dryden Road, Wonford, Exeter (formerly Registrar, Langdon Hospital, Dawlish, Devon)

Over recent years attention has been directed towards providing appropriate facilities for the mentally abnormal offender. In the past such individuals were probably absorbed into the hospital system. However, the open door policy of the 1960s and 1970s, together with hospital closures and reduced bed numbers, has meant that this invisible absorption has been unable to continue. It has been suggested that many of these mentally abnormal offenders are inappropriately placed within the penal system, thus contributing to prison overcrowding. ${ }^{1.2}$ The Butler and Glancy Reports ${ }^{3,4}$ recommended Regional Secure Units as a possible solution. At the beginning of 1987,14 of these had opened. ${ }^{5.6}$ The tendency has been for Regional Secure Units to deal mainly with the mentally ill offenders and the needs of the mentally impaired have been largely overlooked. The literature on the subject of this latter group has been sparse. Craft ${ }^{7}$ described an isolated unit in Wales, which dealt with mentally abnormal offenders, and there are hospitals which provide secure facilities for the mentally handicapped. ${ }^{8}$ The purpose of this study is to describe the characteristics of the patients admitted to an open forensic unit in order to assess the suitability of the facilities and the outcome of the treatment provided.

\section{Background}

Since 1967, the Leander Unit in Langdon Hospital has developed as a service for the mentally abnormal offenders in Devon and Cornwall. The Unit comprised three villas in a mental handicap hospital and dealt mainly with offenders who functioned in the dull normal range. The Regional Secure Unit (the Butler Clinic) was sited at Langdon Hospital and opened in 1983. Since then the two units have functioned side by side. The Leander Unit has recently been redeveloped and now consists of two wards, each with 21 open beds, a 12-bedded pre-discharge unit and a resource centre, providing a base for psychology, social work, education, occupational therapy and industrial therapy, as the rest of this hospital contracts and closes.

- All wards integrate male and female patients. The admission, assessment and intensive care ward includes a three-bedded secure suite which can be used for the acutely disturbed patient or wandering patient. When ready, patients move onto the rehabilitation ward and, if indicated, the predischarge unit, to develop independent living skills. As the Leander Unit evolved from the mental handicap services, it provides the facilities traditionally found in mental handicap hospitals and emphasises treatment, training and rehabilitation. However, as it functions as part of a wider forensic psychiatric service it offers additional expertise, since the multi-disciplinary team are all experienced at working with forensic patients. It is staffed by registered nurses for the mentally handicapped with a high staff to patient ratio of just less than $1: 1$. The medical staff consists of two consultants, a senior registrar, a registrar and a clinical assistant.

The Unit is seen as a medium-term facility with emphasis on rehabilitation and discharge into appropriate accommodation and employment. Close links with community facilities are encouraged and assisted by the involvement of a liaison probation officer. Community follow up is carried out initially by Leander staff.

\section{The study}

All mentally abnormal offenders already in-patients or admitted to the Unit between 1 January 1984 and 31 December 1986 were included for the study. The 1984 starting date was chosen as this was the first full year that the Regional Secure unit was open. The case notes of these patients were examined and standardised information concerning 60 personal variables was extracted from the Devon and Cornwall Psychiatric Service Computerised Case Register. Diagnoses were assigned according to ICD-9 criteria.

Demographic data There were 50 mentally abnormal offenders treated in the Leander Unit during this three year period. Of these, $48 \%$ were admitted during the study period. Males outnumbered females by nine to one. The average age at the time of referral was 31.4 (range 17-57); $80 \%$ of the patients were single, $14 \%$ divorced, $4 \%$ married and $2 \%$ widowed.

Location on referral People were referred from a wide range of sources. The majority $(56 \%)$ were referred while on remand in prison. A further $18 \%$ came directly from special hospitals. Others were referred from police custody, bail hostels or remand on bail in the community.

Past and present criminal behaviour Only four patients had no previous criminal record and the average number of preconvictions was 6.8 (range $0-65$ ). The average age on first conviction was 22 (range 9-50). Of a total of 76 current offences, 28 were sexual offences, 21 property offences, seven arson and nine crimes of violence; $40 \%$ of the patients had been convicted of more than one current offence. 
Psychiatric diagnosis Of the patients, 27 had no formal psychiatric diagnosis recorded in the notes. Four had a definite diagnosis of schizophrenia, four were manic depressives; a further four were labelled as having a personality disorder, following brain damage. Two were diagnosed as having neurotic depression and one had Huntington's chorea.

An IQ assessment had been had at some time by $80 \%$ of patients and the average IQ was found to be 74 (range 50-98). Eleven patients were found to have an IQ below 70. Of these, ten fulfilled the ICD-9 criteria for mild mental retardation and one for moderate mental retardation. Three patients carried more than one psychiatric diagnosis. Many were also handicapped by chronic physical conditions such as epilepsy (10), hearing impairment (7), brain damage (4) and speech impediment (3).

Previous institutional experience They all had previous institutional experience, $86 \%$ in more than one institution; $78 \%$ had been in prison, $52 \%$ in mental handicap hospitals, $22 \%$ in special hospitals and $18 \%$ in psychiatric hospitals. Some had also spent time in youth custody, detention centres, Borstal, approved school, children's home, adolescent psychiatric units and community hostels.

Problem behaviours All the patients had exhibited some kind of problem behaviour, prior to the index offence, such as sexual misbehaviour, absconding, violence, deliberate self harm, alcohol or drug abuse, fire setting and destruction of property.

Legal status on admission Of the patients, $60 \%$ were detained under the Mental Health Act 1983. Of these, 13 were on Restriction Orders. Nearly all of the remaining patients (32\%) were on Probation Orders (Powers of the Criminal Courts Act 1983, Section 3). There appeared to be no correlation between legal status and psychiatric diagnosis.

Mental Health Act classification of the $\mathbf{3 0}$ patients on Orders of the Mental Health Act, 17 were classified as suffering from mental impairment, seven from mental illness, five from psychopathic disorder and one from severe mental impairment. Those who were admitted under the Mental Health Act, but who had no formal psychiatric diagnosis recorded in the notes, were classified as mentally impaired.

Length of stay in the Leander Unit The average length of stay on the unit was 50 months (range 1-249). However, this was a skewed distribution with 25 of the patients spending less than two years in hospital.

Outcome Twenty-eight patients improved sufficiently to be considered ready for discharge, or the next stage of rehabilitation in the community. One patient absconded, but no follow-up details are available.

Two patients who had been admitted on trial leave from Special Hospitals were transferred back as there appeared to be no change in their sexual behaviour despite treatment. The risk of re-offending was considered to be too high for them to remain in an open setting. Three patients reoffended, within the hospital, while in-patients and were taken into custody. These offences were sexual and physical assaults on other patients.
Ten patients were still in the unit at the end of 1986. Four of these were awaiting community placements, three continued to require treatment and three were considered to require continuing care in hospital, for the foreseeable future. At the end of 1986, the numbers of patients were temporarily low because of redevelopment and upgrading of the unit.

\section{Comment}

As a group, these patients resembled the general criminal population more closely than a psychiatric hospital population in terms of the sex ratio. Males are convicted nine times more commonly than women, which matches the sex ratio of the patients on the unit.

The fact that the vast majority of the patients were single or divorced suggests that they are a group who have diffculty forming and maintaining relationships. The average age on referral was 31 . This is older than would have been expected considering the fact that half of all indictable crimes are committed by males under the age of 21 and that the average age at first conviction is 20 . The older age may be explained by the fact that referral to the unit is often a last resort after all other options have been tried. This is confirmed by the long criminal record which many of the patients had, as well as their invariably long history of institutional experience which often includes prison.

Sexual crimes accounted for $37 \%$ of index offences. These were predominantly indecent assaults and often involved children of both sexes. This contrasts with the fact that in Britain, sexual offences account for less than $1 \%$ of all indictable crimes recorded by the police; $9 \%$ of the index offences were arson, which is also higher than would be expected. It is well recognised that sexual offences and arson are committed more commonly by those in the lower intellectual range. 9.10

Many of the patients had no formal psychiatric diagnosis recorded in their notes. Although only 11 fulfilled the ICD9 criteria for mental retardation, many functioned in the dull normal range and 17 admissions were under the Mental Health Act classification of mental impairment. Considering their histories, it is possible that a number of the patients suffered from a personality disorder. However, this diagnosis was rarely recorded in the notes and cannot be made retrospectively on the basis of case note review alone. Psychiatric diagnosis appeared to be unrelated to the legal status on admission. Those with a formal psychiatric diagnosis were as likely to be admitted on a Probation Order as under the Mental Health Act. The length of time spent in the unit varied enormously. Twenty-five spent less than two years there. Of those who stayed longer, some simply progressed at a slower rate. Others improved considerably, but their discharge was delayed due to lack of facilities in the community and a small group remained unsuitable for life outside hospital because of the risk of re-offending.

The results suggest that these are a group of offenders with manifold problems who defy easy categorisation. Many of the patients have no formal psychiatric diagnosis. It is not surprising, therefore, that they are inadequately 
catered for by the alternative facilities and often end up in the penal system. The majority of the patients are seen as too able for the mental handicap services, which are, in any case, now mainly community based with very few in-patient beds. The general psychiatric services also tend to reject this group because they are considered to be too dull and often not mentally ill. The often emotive nature of their offending behaviour, e.g. sexual offences against children and arson, further deters the general psychiatric teams. However, their behaviour rarely justifies the levels of security offered by Special Hospitals or Regional Secure Units.

The encouraging results and low incidence of re-offending or unacceptable behaviour as in-patients suggests that there is a need for a specialised service to cater for the type of offender described. A unit, such as the one described, provides staff who are experienced at dealing with this group, as well as a higher staff to patient ratio than is usual. As a result difficult behaviour, which is too disruptive to be tolerated in other open facilities, can be more easily contained or prevented. Having these patients who have much in common concentrated in one unit allows the further development of staff expertise.

Apart from the hospital provisions discussed above, the alternative to such a unit would be some kind of communitybased service, such as a staffed hostel. The majority of the patients admitted to the Leander Unit are referred because they have been unable to live in the community, sometimes in hostels, despite receiving much support. This suggests a need for in-patient treatment. Smaller community units, or non-specialist units are also unable, either on practical or economic grounds, to offer the wide range of services and facilities provided by an in-patient forensic unit which is part of a wider forensic service.

The need for a health service provision at all might be debated particularly on the grounds that there is often no clear psychiatric diagnosis. However, the group described have obvious deficits, and handicaps which respond to treatment and training. On this basis it seems preferable that they are treated in hospital rather than allowing them to drift into the penal system. As far as the public's safety is concerned, unless such individuals are imprisoned for life, the safest option must be a flexible system, which provides treatment,. community support and follow-up, together with the facility for easy readmission to hospital, if necessary. Cost effectiveness is difficult to assess accurately. However, the cost of running the unit has to be set against that of keeping these individuals inappropriately in other facilities.

The main limitation of the current forensic service described here is the lack of appropriate community provisions to move patients on to when they are ready for discharge. This means that the Unit's throughput is slower than it should be. It is, however, becoming apparent that for a small group of patients there is a need for long-term facilities within a well-supervised, protected environment, probably a hospital ward, as their behaviour continues to make them unacceptable to the public at large. As yet, the numbers requiring such a provision have not been established.

The data available so far suggest that the majority of patients admitted to the Leander Unit benefit significantly and go on to live in the community. The results also imply that it is possible to run an open forensic unit with only a very low risk of absconding and re-offending, provided it is well staffed. Longer term follow-up studies would be needed in order to confirm this.

Langdon Hospital is possibly unique in providing an open forensic unit for this group on the same site as the Regional Secure Unit. This enables the sharing of expertise and facilities, together with the development of a comprehensive forensic service.

With the continuing reduction in psychiatric and mental handicap hospital beds, together with mounting pressure on the prison service, the need for facilities catering for this group of offenders is likely to increase. The Unit described here offers a possible solution for this difficult-to-place group.

\section{ACKNOWLEDGEMENTS}

I am grateful to Drs Martin Donovan and Arden Tomison for their valuable comments on this paper. I would also like to thank Sue Sullivan for her secretarial help.

\section{REFERENCES}

${ }^{1}$ Penrose, L. S. (1939) Mental disease and crime. Outline of a comparative study of European statistics. British Journal of Medical Psychology, 18, 1-15.

${ }^{2}$ LeGAL CoRrespondent (1985) Mentally ill offenders in prison. British Medical Journal, 290, 447.

${ }^{3}$ Department of Health and Social Security and the Home OFfice (1975) Report of the Committee on Mentally Abnormal Offenders (Butler Report) (1975) London: HMSO.

4Working Party on Security in NHS Hospitals (1974) Revised Report (Glancy Report). London: Department of Health \& Social Security.

${ }^{5}$ SNOWDEN, P. (1985) A survey of a Regional Secure Unit programme. The British Journal of Psychiatry, 147, 499-507.

6 - (1987) Regional Secure Units: arriving but under threat. British Medical Journal, 294, 1310-1311.

'CRAFT, M. (1984) Should one treat or gaol psychopaths? In Mentally Abnormal Offenders (eds M. \& A. Craft). London: Baillière Tindall.

${ }^{8}$ ISWERAN, M. S. \& BARDSLeY, E. M. (1987) Secure facilities for mentally impaired patients. Bulletin of the Royal College of Psychiatrists, 11, 52-54.

${ }^{9}$ PowER, D. J. (1969) Subnormality and crime. Medicine, Science and the Law, 9, 82-93 and 162-171.

${ }^{10}$ ReID, A. M. (1982) The Psychiatry of Mental Handicap. Oxford: Blackwell Scientific. 